Instrumental Achievements

\title{
Capillary Gas Chromatography of Saturated Hydrocarbons in Gasoline by Removing Unsaturated Components by Precolumn Sulfonation
}

\author{
Koichi Shomi and Seiji TAKahashI \\ Kyoto Analytical Application Laboratory, Shimadzu Corporation, \\ Nishinokyo-Kuwaharacho, Kyoto 604
}

The paraffin, olefin, naphthene and aromatic (PONA) analysis in gasoline or naphtha has long been carried out by separating the constituent hydrocarbons (HC) with a capillary column having a nonpolar stationary phase, into as many peaks as possible, followed by the peak identification and the quantification as groups.

The PONA analysis technique often encounters a serious problem in identifying hundreds of peaks obtained in a run. Even the gas chromatography/mass spectrometry (GC/MS) combined technique, which is now believed to be one of the most reliable methods for qualitative determination, can often give erroneous qualitative information on unresolved GC fractions. When the GC/MS combined technique cannot be utilized for the qualitative determination of GC fractions, it is necessary to identify GC peaks from their retention times by comparing them with those of standard pure compounds. The qualitative errors can be minimized if particular type of hydrocarbons are removed before the sample is chromatographed. We have reported the multidimensional capillary GC method (MDCGC) developed for the above purpose', in which the aromatics in the naphtha samples were selectively removed so that only the paraffin and naphthene peaks were recorded. The present paper refers to our experiments carried out to improve the method. Just after a gasoline sample was injected into the GC apparatus, the olefin and aromatic constituents were removed through sulfonation, so that only paraffins and naphthenes were detected on the chromatogram. Comparison of the chromatograms before and after the sulfonation makes it possible to discriminate saturated HC fractions from unsaturated ones, and to see whether a saturated $\mathrm{HC}$ and an unsaturated one are eluted without being separated.

\section{Experimental}

Instruments and operating conditions are listed in Table 1 .

The agent to trap and remove unsaturated HCs (olefins and aromatics) was prepared in the following way. Eight grams of water and $2 \mathrm{~g}$ of concentrated sulfuric acid were placed in a shallow container and mixed well. Five grams of Shimalite diatomaceous

earth solid support (80-100 mesh) was added to this solution, and dried at $100^{\circ} \mathrm{C}$ in an oven with occasional mixing.

It is necessary to prevent sulfuric acid vapor from entering the capillary column because it would deteriorate the column. The trapping agent used for this purpose was prepared in the same way as above. Eight grams of water and $2 \mathrm{~g}$ of $\mathrm{KOH}$ were placed in a shallow container and mixed well. Five grams of Shimalite diatomaceous earth solid support (80-100 mesh) was added to this solution, and dried at $100^{\circ} \mathrm{C}$ in an oven with occasional mixing.

The addition type reaction of sulfuric acid to olefins and aromatics proceeds rather slowly, if the sulfuric acid contains some water. In order to accelerate the reaction, the trapping agents of unsaturated HCs were dried in the following way. In a glass tube, $2.6 \mathrm{~mm}$ i. $d$. and $13 \mathrm{~cm}$ long, the trapping agent for unsaturated HCs was packed to a length of $3 \mathrm{~cm}$ and that for

Table 1 Instruments and operating conditions

Gas chromatograph

Attachment

Data processor Column

GC/MS system

Operating conditions Column temp.

Injection temp.

Detector temp.

Detector

Carrier gas

Split ratio

Septum purge flow

Sample size
Shimadzu GC-15APFsc (dedicated to capillary GC and equipped with a temperature-controlled carrier gas flow control unit) CRG-15 cryogenic work attachment

Shimadzu C-R3A Chromatopac Shimadzu HiCap CBP-1M50-025 (chemically bonded fused silica capillary column with dimethyl silicone stationary phase, $50 \mathrm{~m}$ long, $0.22 \mathrm{~mm}$ i.d., and $0.25 \mu \mathrm{m}$ in film thickness)

Shimadzu GCMS-QP1000

$0^{\circ} \mathrm{C}\left(5 \mathrm{~min}\right.$ hold) to $120^{\circ} \mathrm{C}$ at $3^{\circ} \mathrm{C} / \mathrm{min}$

$120^{\circ} \mathrm{C}$

$200^{\circ} \mathrm{C}$

FID

He $2 \mathrm{~kg} / \mathrm{cm}^{2}, 25 \mathrm{~cm} / \mathrm{s}$

$1 / 300$

$10 \mathrm{ml} / \mathrm{min}$

$0.5 \mu \mathrm{l}$ 


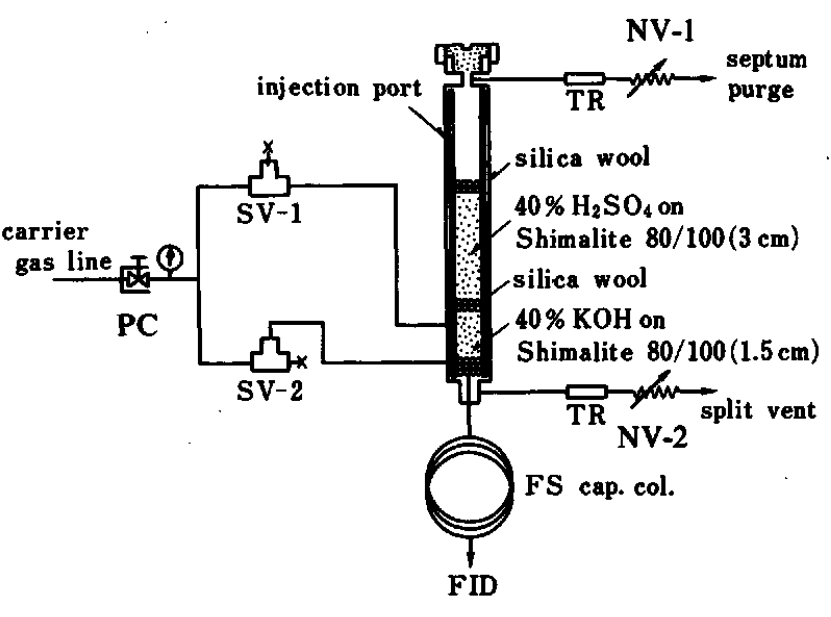

Fig. 1 Flow diagram of precolumn sulfonation device. sulfuric acid vapor to a length of $1.5 \mathrm{~cm}$, as shown in Fig. 1. This glass tube was connected to the sample injection port of the GC, with the trapping agent for sulfuric acid vapor at the upstream position. Then, the conditioning was carried out at $120^{\circ} \mathrm{C}$ with carrier gas flow for one hour.

The trapping tube described before is installed to the GC apparatus in the way shown in Fig. 1. All the parts shown in Fig. 1-pressure control valve (PC), solenoid valves (SV-1 and SV-2), molecular sieve traps (TR), and needle valves (NV-1 and NV-2)-are included in the Grob type split/splitless sample injector which is equipped with the standard GC-15APFsc gas chromatograph. The only difference is the connection of the carrier gas line to the solenoid valve. ${ }^{1}$

The operation is made as follows.

i) Open the solenoid valve SV-1, close the solenoid valve SV-2, and allow the carrier gas to flow. The carrier gas flows into the top of the trapping tube and

Table 2 Saturated and aromatic hydrocarbons identified in the gasoline sample

\begin{tabular}{|c|c|c|c|c|c|c|c|}
\hline Peak & Component name & Peak & Component name & Peak * & Component name & Peak & Component name \\
\hline 1 & iC4 & 35 & 223TMC5 & 69 & $3 \mathrm{EC} 8$ & (1) & B \\
\hline 2 & $\mathrm{nC4}$ & 36 & 112TMCyC5 & 70 & $2 \mathrm{MC} 9$ & (2) & To1 \\
\hline 3 & iCs & 37 & 23DMC7 & 71 & $4 \mathrm{MC} 9$ & (3) & EB \\
\hline 4 & $\mathrm{nC5}$ & 38 & $2 \mathrm{MC7}$ & 72 & $3 \mathrm{MC9}$ & (4) & pX \\
\hline 5 & 22DMC4 & 39 & $4 \mathrm{MC7}$ & 73 & $\mathrm{nC10}$ & (5) & $\mathbf{m X}$ \\
\hline 6 & CyC5 & 40 & 13DMCyC6 & 74 & 5E2MC8 & (6) & oX \\
\hline 7 & 23DMC4 & 41 & $3 \mathrm{MC7}$ & 75 & 25DMC9 & (7) & iPB \\
\hline 8 & $2 \mathrm{MC5}$ & 42 & 1Mt3ECyC5 & 76 & $26 \mathrm{DMC} 9$ & (8) & nPB \\
\hline 9 & $3 \mathrm{MC5}$ & 43 & 1Mc3ECyC5 & 77 & 23DMC9 & (9) & mETo1 \\
\hline 10 & nC6 & 44 & 1M2t2ECyC5 & 78 & $5 \mathrm{MC10}$ & (10) & pETo1 \\
\hline 11 & MCyC5 & 45 & 1t2DMC5 & 79 & 3EC9 & (11) & 135TMB \\
\hline 12 & 24DMC5 & 46 & 1c2DMCyC6 & 80 & $2 \mathrm{MC10}$ & (12) & oETo1 \\
\hline 13 & 22DMC5 & 47 & $\mathrm{nC8}$ & 81 & $3 \mathrm{MC10}$ & (13) & 124TMB \\
\hline 14 & 23DMC5 & 48 & 11c3t4TMCyC5 & 82 & $\mathrm{nC11}$ & (14) & iPB \\
\hline 15 & 33DMC5 & 49 & 1133TeMCyC5 & 83 & $5 \mathrm{MC11}$ & (11) & 123TMB \\
\hline 16 & CyC6 & 50 & $1 \mathrm{M} 3 \mathrm{ECyC5}$ & 84 & $3 \mathrm{EC} 10$ & (16) & 1MiPB \\
\hline 17 & 2MC6 & 51 & 224TMC6 & 85 & $2 \mathrm{MC11}$ & (17) & 1M4iPB \\
\hline 18 & $3 \mathrm{MC6}$ & 52 & MCyC7 & 86 & $3 \mathrm{MC11}$ & (18) & INDANE \\
\hline 19 & 1t3DMCyC5 & 53 & ECyC6 & 87 & $\mathrm{nC12}$ & (19) & 13DEB \\
\hline 20 & 1c3DMCyC5 & 54 & 25DMC7 & & & (20) & 1M2nPB \\
\hline 21 & 1t2DMCyC5 & 55 & C9N & & & (21) & 13DM5EB \\
\hline 22 & $3 E C 5$ & 56 & 1c3c5TMCyC6 & & & (22) & $\mathbf{n B B}$ \\
\hline 23 & 224TMC5 & 57 & 135TMCyC6 & & & (20) & 1M2nPB \\
\hline 24 & $\mathrm{nC7}$ & 58 & 24DMC7 & & & (24) & 14DM2EB \\
\hline 25 & MCyC6 & 59 & 4EC7 & & & (25) & 13DM4EB \\
\hline 26 & 113TMCyC5 & 60 & $2 \mathrm{MC} 8$ & & & (26) & 12DM4EB \\
\hline 27 & 22DMC6 & 61 & $4 \mathrm{MC8}$ & & & (27) & 12DM3EB \\
\hline 28 & ECyC5 & 62 & 113TMC6 & & & (28) & 13DM2EB \\
\hline 29 & 25DMC6 & 63 & $3 \mathrm{MC8}$ & & & (20) & 1245TeMB \\
\hline 30 & 24DMC6 & 64 & 1M3ECyC6 & & & (30) & $1235 \mathrm{TeMB}$ \\
\hline 31 & 1c2c4TMCyC5 & 65 & $\mathrm{nC9}$ & & & (31) & 7MINDANE \\
\hline 32 & 33DMC6 & 66 & nC3CyC6 & & & (32) & $1 \mathrm{M} 4 \mathrm{nBB}$ \\
\hline 33 & 123TMCуC5 & 67 & $36 \mathrm{DMC} 8$ & & & (3) & $1234 \mathrm{TeMB}$ \\
\hline 34 & 234TMC5 & 68 & 5MC9 & & & (34) & NAPHTHALENE \\
\hline
\end{tabular}

i, iso; n; normal; D, di; T, tri; Te, tetra; M, methyl; E, ethyl; P, propyl; B, butyl; c, cis; t, trans; Cy, cyclo; Tol, toluene; $\mathrm{X}$, xylene; o, ortho; $\mathrm{m}$, meta; $\mathrm{p}$, para; $\mathrm{N}$, naphthene. $\mathrm{C4}$, butane, $\mathrm{C} 5$, pentane; $\mathrm{C6}$, hexane; $\mathrm{C} 7$, heptane; $\mathrm{C}$, octane; $\mathrm{C}$, nonane; $\mathrm{C10}$, decane; $\mathrm{C} 11$, undecane; $\mathrm{C12}$, dodecane. 

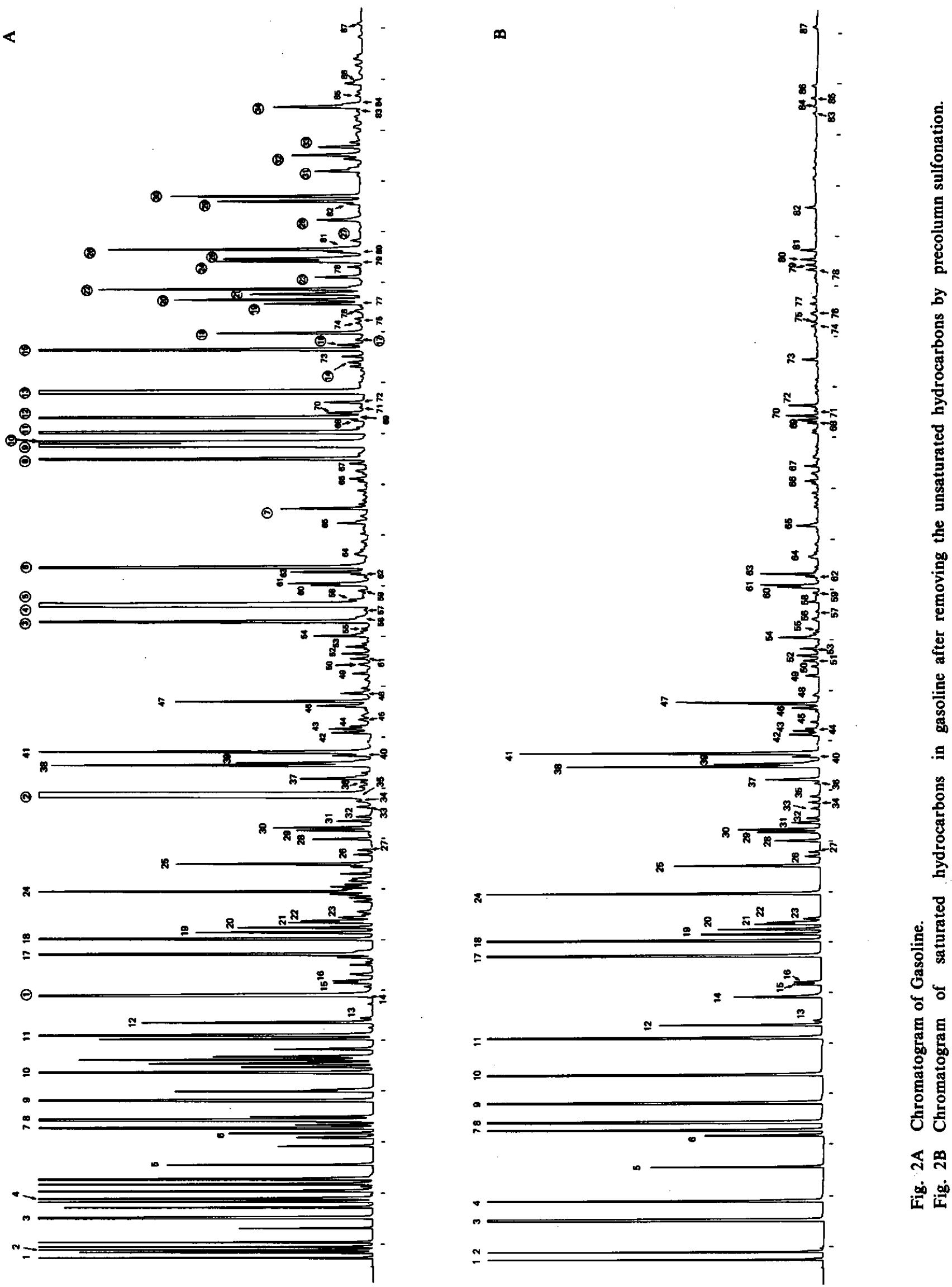
toward the capillary column, in the same way as in the ordinary capillary GC. The flow rate in the septum purge line is $10 \mathrm{ml} / \mathrm{min}$ and that at the split vent is 300 $\mathrm{ml} / \mathrm{min}$. Cool the column oven to $0^{\circ} \mathrm{C}$ and inject $0.5 \mu \mathrm{l}$ of the sample.

ii) At $30 \mathrm{~s}$ after sample injection, close the solenoid valve $S V-1$ and open the solenoid valve $S V-2$. The carrier gas flows through the SV-2 and enters the trapping tube from the bottom and flows toward the capillary column. At the same time, the carrier gas flows upward through the trapping tube and prevents the products formed in sulfonation and the sulfuric acid vapor from flowing into the capillary column.

iii)Determination of sulfonation temperature: The sulfonation which is a gas-phase reaction must occur instantaneously. Our experiment showed that olefins were sulfonated instantaneously at $80^{\circ} \mathrm{C}$ but that aromatics were not sulfonated rapidly enough; especially the sulfonation of benzene required the higher temperature of $120^{\circ} \mathrm{C}$. The $3 \mathrm{~cm}$ long trapping agent can be used up to 10 injections of $0.5 \mu l$ gasoline sample.

iv) The analysis of the orginal gasoline samples was carried out using the same flow line, with its trapping tube replaced with an empty glass insert. The other operational conditions were the same as mentioned above.

\section{Results and Discussion}

Figure 2A shows a chromatogram of a gasoline sample from which no components have been removed, and Fig. 2B shows that of a sample from which olefins and aromatics have been removed. Those peaks that are found in Fig. 2A but not in Fig. 2B correspond to unsaturated $\mathrm{HC}$ fractions. The saturated HCs were identified with the Shimadzu GCMS-QP1000 and through retention time comparison using pure standard compounds. Table 2 shows the results of qualitative determination of the main saturated HCs and aromatic HCs.

Comparison of the Fig. 2A and Fig. 2B shows:

i) Olefins are eluted close to $n$-paraffins of the same number of carbon atoms.

ii) A series of branched paraffins are eluted before corresponding $n$-paraffins in a certain order, for example, peak \#38-41 before \#47 (n-octane), peak $\# 60$ - 63 before \#65 ( $n$-nonane), peak $\# 68-72$ before $\# 73$ ( $n$-decane), peak \#78-81 before \#82 ( $n$-undecane), peak \#83-86 before \#87 ( $n$-dodecane).

iii) Contents of olefins etuted after $\boldsymbol{n}$-octane are minor.

iV) Comparison of peak heights in Fig. 2A and Fig. 2B shows that 1-trans-3-dimethyl-cyclopentane (peak \#19), 2,2,4-trimethylpentane (peak \#23), 1-methyl-cis-3ethylcyclopentane (peak \#43) 1-cis-2-dimethylcyclohexane (peak \#46), 1,1-cis-3-trans-4-trimethylcyclopentane (peak \#48) are co-eluted with olefin fractions.

\section{Reference}

1. K. Shiomi, Anal. Sci, 1, 327 (1985).

(Received April 23, 1987)

(Accepted May 25, 1987) 